\title{
Correlation between Laser Fluorescence Readings and Volume of Tooth Preparation in Incipient Occlusal Caries In Vitro
}

\author{
EDUARDO S. GHANAME, DDS, MS* \\ ANDRÉ V. RITTER, DDS, MS ${ }^{\dagger}$ \\ HARALD O. HEYMANN, DDS, MEd ${ }^{\ddagger}$ \\ WILLIAM F. VANN, Jr., DMD, MS, PHD \\ DANIEL A. SHUGARS, DDS, MPH, PHDף \\ JAMES D. BADER, DDS, MPH**
}

\begin{abstract}
Objective: This study evaluated the correlation between laser fluorescence readings and the extent of incipient occlusal caries as measured by the volume of tooth preparation in vitro.

Materials and Methods: One hundred and three permanent molars and premolars containing incipient occlusal pit-and-fissure caries and sound occlusal surfaces (1/4 of the sample, control) were selected. DIAGNOdent (KaVo Dental Corporation, Lake Zurich, IL, USA) readings were obtained according to manufacturer instructions. Caries was removed with $1 / 4$ round burs in high speed. The volume of tooth preparation was measured using a surrogate measure based on the amount of composite needed to fill the preparations. Sensitivity and specificity using different cutoff values were calculated for lesions/preparations extending into dentin.

The results were analyzed statistically.

Results: The Pearson correlation for preparation volume and DIAGNOdent reading measurements was low $(r=0.285)$. Sensitivity and specificity of DIAGNOdent for detection of dentinal lesions were 0.83 and 0.60 , and 0.66 and 0.73 for the cutoff values of 20 and 30, respectively. Conclusions: Within the limitations of this study, laser fluorescence measured with DIAGNOdent does not correlate well with extent of carious tooth structure in incipient occlusal caries.
\end{abstract}

\section{CLINICAL SIGNIFICANCE}

Clinicians should not rely only on DIAGNOdent readings to determine the extension of incipient occlusal caries.

(J Esthet Restor Dent 22:31-41, 2010)

*Graduate student, Department of Cariology, Restorative Sciences, and Endodontics, University of Michigan, Ann Arbor, MI, USA ${ }^{\dagger}$ Associate professor, Department of Operative Dentistry, University of North Carolina, Chapel Hill, NC, USA ${ }^{\ddagger}$ Professor and graduate program director, Department of Operative Dentistry, University of North Carolina, Chapel Hill, NC, USA ${ }^{\S}$ Distinguished professor, Department of Pediatric Dentistry, University of North Carolina, Chapel Hill, NC, USA

"Professor, Department of Operative Dentistry, University of North Carolina, Chapel Hill, NC, USA *Research professor, Department of Operative Dentistry, University of North Carolina, Chapel Hill, NC, USA 
INTRODUCTION

$\mathrm{S}_{\mathrm{o}}^{\mathrm{u}}$ uccessful management of occlusal caries lesions requires an accurate detection of their presence, dimension, and activity status. ${ }^{1}$ Common methods used by dental practitioners to detect caries lesions are based on visual, tactile, and radiographic examinations., ${ }^{2,3}$ Because these methods are highly dependent on subjective interpretation, discrepancies among dentists' diagnoses tend to be frequent, especially when diagnosing incipient lesions. ${ }^{4,5}$ In a systematic review of the literature, Bader et al. ${ }^{6}$ reported that sensitivity and specificity of caries detection vary greatly for visual, visual-tactile, and radiographic diagnostic methods. The authors concluded that "the available information is insufficient to support generalizable estimates of the sensitivity and specificity of any given application of a diagnostic method."

It would be beneficial for clinicians to have objective methods for caries diagnosis and caries lesion detection. Several innovative methods for detecting caries lesions have recently become available, including measurements of the scattering of light, fiber-optic transillumination, ultrasound imaging, electrical conductance measurements, and laser fluorescence. ${ }^{7}$
DIAGNOdent (KaVo Dental Corporation, Lake Zurich, IL, USA) is a small, lightweight, battery-powered, chairside, handheld instrument that measures laser fluorescence within the tooth structure. The instrument is intended to detect cavitated and non-cavitated occlusal and smooth surface caries. ${ }^{8}$ The unit operates at a wavelength of $655 \mathrm{~nm}$ and generates a red laser light that is directed to the tooth structure by a probe. As the laser light is propagated into the tooth, two-way handpiece optics permit the unit to quantify the reflected laser light energy. According to the manufacturer, at this specific wavelength, a healthy non-carious tooth structure exhibits little or no fluorescence, resulting in low scale readings on the monitor, whereas a carious tooth structure exhibits degrees of fluorescence that result in elevated scale readings on the DIAGNOdent monitor. ${ }^{9}$ The instrument produces DIAGNOdent values between 0 and 99. According to the manufacturer, these values correlate with presence/absence of enamel and dentin caries.

The manufacturer promotes the instrument as an objective diagnostic aid to help clinicians detect caries at the earliest possible stage. Although DIAGNOdent has been validated in in vitro ${ }^{10-12}$ and in vivo $^{13,14}$ studies, there is limited and inconclusive scientific evidence to support a direct correlation between laser fluorescence readings and the extent of caries lesions.

Heinrich-Weltzien et al. ${ }^{15}$ suggested that increasing DIAGNOdent readings are correlated with increasing caries lesion depth, at least up to a reading of 34 . The clinical implication would be that clinicians can rely on the laser fluorescence readings not only as a detection tool but also to track the growth of a caries lesion over time. Other reports indicate that high DIAGNOdent reading values do not correlate positively with depth of caries lesions. ${ }^{16-18}$ One study conducted in 25 patients receiving minimally invasive restorations for incipient occlusal lesions found no significant correlation between preparation volume and mean, maximum, or minimum peak DIAGNOdent reading. ${ }^{18}$ Considering the small sample size (only 32 teeth were non-cavitated), the potential confounding effect of staining $(90 \%$ of the teeth had darkly stained pits and fissures), and the inherent difficulty of obtaining precise measurements in vivo, there remains a need to verify the correlation between laser fluorescence readings and the volume of tooth preparations under more standardized conditions.

Lussi et al. ${ }^{2}$ established optimal cutoff values with specific treatment recommendations: no active 
treatment for DIAGNOdent readings between 0 and 15 , preventive measures or operative treatment depending on patient caries risk for readings between 16 and 30, and preventive and operative treatment for readings 31 and over. However, the usefulness of such recommendations is highly dependent on the caries detection performance of the instrument in use. A systematic review of the DIAGNOdent performance for detecting caries indicated that although the instrument is more sensitive than traditional diagnostic methods such as visual assessment and bitewing radiographs, its low specificity makes it a poor primary detection device. ${ }^{19}$

In view of the increasing interest in the development of techniques to augment the detection accuracy of visual, tactile, and radiographic dental caries examinations, and of the limited and inconclusive research available in this area, the aim of this study was to evaluate the correlation between laser fluorescence readings (as measured by DIAGNOdent) and the extent of incipient occlusal carious tooth structure as measured by the volume of tooth preparation in vitro. The study examined the null hypothesis that laser fluorescence readings have no correlation with the amount of tooth structure removed during tooth preparation in incipient occlusal caries in vitro.
In addition, this study aimed to determine the sensitivity and specificity of DIAGNOdent for identifying lesions in dentin.

\section{MATERIALS AND METHODS}

One hundred and three permanent human molars and premolars with occlusal surfaces ranging from sound to localized enamel breakdown (the International Caries Detection and Assessment System [ICDAS] pit-and-fissure codes $0-3)^{20}$ were collected from dental clinics at the University of North Carolina School of Dentistry. The sample included approximately equal number of specimens with ICDAS codes $0,1,2$, and 3 . Specimens were not examined intraorally prior to extraction. All soft tissues and periodontal tissues were removed gently with hand instruments. All specimens were mounted on specimen holders and were stored in water throughout the duration of the study. Disinfection of the specimens was avoided to prevent confounding effects on DIAGNOdent readings. ${ }^{21}$

The occlusal surface of all specimens was photographed with a digital camera at $4 \times$ magnification.

\section{Laser Fluorescence Readings}

The DIAGNOdent instrument was calibrated on a porcelain standard provided by the manufacturer.

Before each use, the instrument was also calibrated for each specimen just prior to the initial reading. DIAGNOdent readings were obtained according to manufacturer's specifications. Specimens were removed from the storage medium and briefly dried prior to each reading. The occlusal surfaces were treated with air-driven particle abrasion (prophylaxis) using PROPHYpearls (KaVo). The pitand-fissure surfaces of each specimen were scanned with the probe perpendicular to the tooth surface, as recommended by the manufacturer. One trained examiner obtained two sets of DIAGNOdent readings. As per the instrument's user guide, the maximum DIAGNOdent value for the entire surface examined ("peak") of each specimen was used in this study. The mean value per tooth was used as the final maximum value for each specimen.

\section{Tooth Preparation}

An initial impression of the occlusal aspect of the specimen was obtained with a clear polyvinylsiloxane impression material (Affinity Crystal, Clinician's Choice, Brookfield, CA, USA) (Figure 1). The incipient occlusal caries lesion was removed by using $1 / 4$ round burs in high-speed handpiece with copious air-water spray. All preparations were completed under operatory light and with the aid of magnification lenses by one trained operator $(2.5 \times$ 

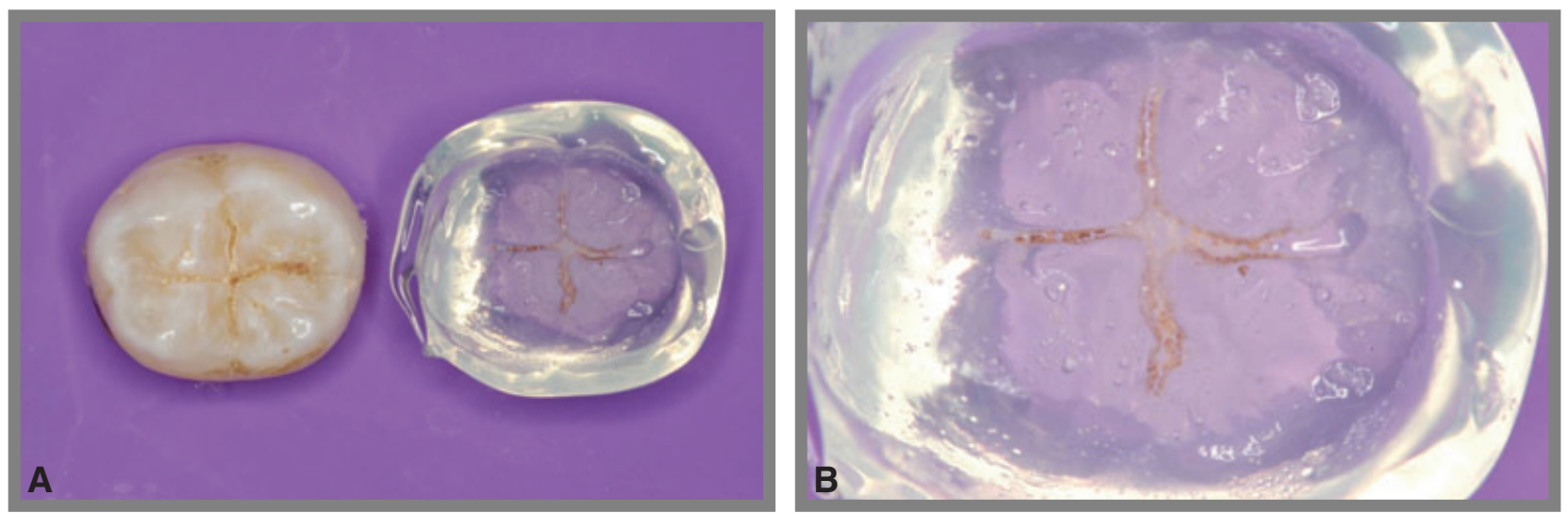

Figure 1. A, Initial impression of the occlusal aspect of a representative specimen used in this study. B, Magnified image of the preoperative occlusal impression.
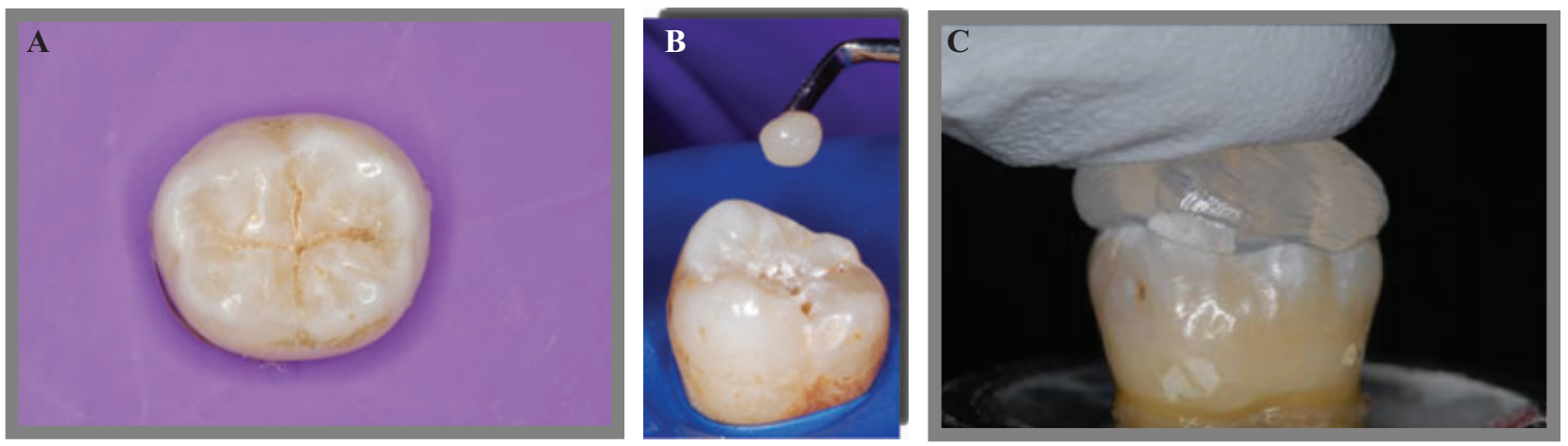

Figure 2. Volume measurement. A, Occlusal view of a specimen used in this study. B, Composite resin being placed into tooth preparation. C, Preoperative occlusal impression being positioned.

magnification). No mechanical retention or resistance form was performed. Visual criteria with the aid of tactile information (dental explorer) were used to determine if all caries/stains had been removed. ${ }^{22,23}$ Preparations that extended into dentin were identified using visual criteria of color change from the adjacent enamel.

\section{Lesion Volume and} Extension Determination

The amount of tooth structure removed during preparation (the cavity volume) was quantified as follows: composite resin (Amelogen Plus, Ultradent, South Jordan, UT, USA) was placed in the preparation with no bonding agent. Care was taken to avoid internal voids and to add composite just enough to completely fill the preparation. The initial occlusal impression was then repositioned on the occlusal surface to establish original form, as an occlusal index (Figure 2). The index was removed, and after the excess composite was carved, the uncured composite resin was removed from the preparation with a dental explorer and weighed on a digital scale (Mettler Toledo, 
Polaris Parkway, Columbus, $\mathrm{OH}$, USA). The volume of the tooth preparation was obtained by multiplying the value of the composite's final weight by its density

$\left(2.1317 \mathrm{~g} / \mathrm{mm}^{3}\right)$. This process was repeated three times, and the mean value was used as the final measure of volume.

After the volume was obtained, the specimens were evaluated carefully under operatory light and magnifying glasses $(4 \times)$ to establish if the end point of each preparation had reached dentin or not. This visual qualification of the preparation's depth allowed the construction of $2 \times 2$ frequency tables as well as the calculation of sensitivity and specificity values of laser fluorescence readings for the detection of dentinal lesions on occlusal surfaces.

\section{Statistical Analysis}

The data were analyzed using SAS statistical software (SAS Institute Inc., Cary, NC, USA). The relationship between the independent variable (DIAGNOdent readings) and the dependent variable (volume) was examined using Pearson correlation. For this analysis, the mean of the two DIAGNOdent readings and the mean volume value were used. A significance level of 0.05 was used for all tests. Specificity and sensitivity of DIAGNOdent for the detection of dentinal lesions at two score cutoffs, 20 and

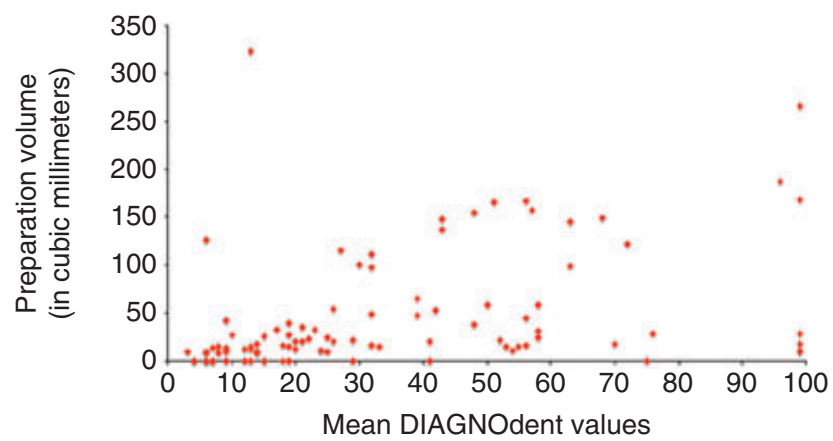

Figure 3. Correlation between DIAGNOdent reading and the preparation volume $(\mathrm{r}=0.285)$.

\begin{tabular}{|c|c|c|c|}
\hline \multirow{2}{*}{$\begin{array}{l}\text { DIAGNOdent reading } \\
\text { (cutoff }=20 \text { ) }\end{array}$} & \multicolumn{2}{|c|}{ Lesion extension } & \multirow[t]{2}{*}{ Total } \\
\hline & In dentin & Not in dentin & \\
\hline Above cutoff & 25 & 29 & 54 \\
\hline Below cutoff & 5 & 44 & 49 \\
\hline Total & 30 & 73 & 103 \\
\hline
\end{tabular}

30, were determined from frequency tables.

RESULTS

A weak correlation was observed when comparing the mean DIAGNOdent values and the volume of tooth preparation $(r=0.285, p>0.05)$. A scatter plot graphic illustrating the relationships observed in this study is depicted in Figure 3.

The frequency of DIAGNOdent readings and their relationship with the extent of the lesion into dentin are presented in Tables 1 and 2. Thirty-three percent of the readings ranged between
0 and $14,11 \%$ ranged between 15 and 20,10\% ranged between 21 and 30 , and $46 \%$ were above 30 .

The sensitivity and specificity for DIAGNOdent were determined for both cutoff values of 20 (initial caries lesion on dentin according to the manufacturer) and 30 (advanced caries lesion in dentin according to the manufacturer). These results are illustrated in Tables 1 and 2. Sensitivity and specificity of DIAGNOdent for the detection of dentinal lesions on occlusal surfaces were calculated and identified at 0.83 and 0.60 for the cutoff value of 20 , and at 0.67 


\begin{tabular}{|c|c|c|c|}
\hline \multirow{2}{*}{$\begin{array}{l}\text { DIAGNOdent reading } \\
\text { (cutoff }=\mathbf{3 0} \text { ) }\end{array}$} & \multicolumn{2}{|c|}{ Lesion extension } & \multirow[t]{2}{*}{ Total } \\
\hline & In dentin & Not in dentin & \\
\hline Above cutoff & 20 & 20 & 40 \\
\hline Below cutoff & 10 & 53 & 63 \\
\hline Total & 30 & 73 & 103 \\
\hline
\end{tabular}

and 0.73 for the cutoff value of 30 , respectively.

\section{DISCUSSION}

Clinicians are continuously searching for better and more accurate methods of caries detection. Currently, the most common methods used by clinicians to detect dental caries are based on visual examination, a combination of visual examination and tactile information, and radiographic assessments. The major concern with these traditional methods is that they are highly subjective, and discrepancies among clinicians' diagnoses tend to occur. The concept of an instrument that can accurately identify the various stages of the caries process and simultaneously quantify the extension of the lesion and offer ideal treatment options is extremely appealing.

Several new methods for caries detection that claim to be more objective, valid, precise, reproducible, and simple to use than traditional visual-tactile-radiographic methods have been introduced in the past decade. One such method is the laser fluorescence instrument DIAGNOdent, intended to afford and smooth surface caries, providing quantification of the caries lesion by a simple numerical index. Furthermore, manufacturer guidelines for the use of DIAGNOdent also offer treatment strategies according to the numerical readings. The advent of this new diagclinical dilemma. Can the clinician rely solely on DIAGNOdent measurements? Does DIAGNOdent provide a more accurate diagnosis than visual examination alone? Furthermore, can the clinician always apply his or her therapeutic recommendations with conviction? The results of this study suggest a small to moderate correlation between laser fluorescence readings (as measured by DIAGNOdent) and the volume of tooth preparation as a surrogate measure of the extent of occlusal caries lesions.

One hundred and three extracted teeth were used in this study. an objective detection of occlusal nostic instrument poses a serious
Storage solutions such as chloramine, formalin, and thymol may have significant influence on the fluorescence measured by DIAGNOdent. ${ }^{21}$ Saliva contains a variety of electrolytes including sodium, potassium, magnesium, calcium, bicarbonate, and phosphates. ${ }^{24}$ To preserve the presence of these electrolytes, and inhibit any influence on the measurement of fluorescence, the teeth were stored in water prior to visual and radiographic assessments and laser fluorescence measurements.

Once the diagnostic tests were performed, the suspected incipient occlusal caries lesion was removed by using $1 / 4$ round burs to provide the most conservative preparation. No mechanical retention or resistance form was used. A combination of visual and tactile criteria was used to determine if all caries/ stains had been removed. Although these end point criteria may be considered subjective, this is the most common method used by clinicians in the completion of caries excavation.

Following tooth preparation, the amount of tooth structure removed was quantified by using composite resin. Other methods for volume quantification were considered, such as the use of dental wax, impression materials, computed tomography, pycnometer, and water displacement. However, the 
last three techniques were discarded because their use in human subjects would be impractical. Volume determination with impression materials was not utilized because of technique sensitivity based on a pilot study (i.e., presence of voids and distortion). In addition, the use of dental wax can be very cumbersome, particularly when dealing with retentive preparations, and therefore, it was not utilized in this study.

Volume determination with composite was completed by packing the resin into the preparation. A preoperative occlusal impression was positioned in order to establish original form. Special care was taken in order to verify the correct position of the preoperative impressions. The lightest possible shade of the composite resin was chosen in order to facilitate the distinction between composite resin and tooth structure during excess removal. However, although the accurate distinction between composite resin and tooth structure was not difficult, the use of dyes may be recommended for easier evaluations.

Volume determination was performed three times per specimen, and the mean value per specimen was used for statistical analysis. The precision of the method was considered satisfactory as the standard deviation for withinspecimen measurements was very small. However, the accuracy of the method might be better evaluated after comparison with other methods such as computed tomography measurements.

Comparison with previously published results is problematic because the great majority of the studies has only evaluated the correlation between laser fluorescence readings and depth of caries lesions. The reason for evaluating the correlation between laser fluorescence readings and volume instead of depth was because of the fact that volume measurement may be more representative of lesion extension, as it offers a multidimensional perspective.

Nevertheless, the correlation coefficient for volume preparation and laser fluorescence readings, as measured by DIAGNOdent, reported in this study $(r=0.228)$ was similar to the coefficient reported by Ouellet et al. ${ }^{16}$ for DIAGNOdent readings and depth of the caries $(r=0.4438)$ and caries in dentin $(r=0.3809)$. However, the coefficient was somewhat smaller than the values reported by AlwasDanowska et al., ${ }^{17}$ who observed correlation coefficients ranging from 0.48 to 0.53 .

Only one study that evaluated the correlation between volume of tooth preparation and DIAGNOdent readings has been published. ${ }^{18}$
The authors analyzed 48 teeth from 25 patients and reported that the correlation between preparation volume and maximum DIAGNOdent reading was only 0.191. However, the authors used a small sample size (32 teeth without cavitation), and the research design may not have been appropriate for addressing the research question. The authors used a low viscosity polyvinylsiloxane material to quantify the volume of the preparations, but they failed to recognize the limitations of the method, such as the likelihood of voids and distortion.

The weak correlation observed in this study may indicate that the intensity of the fluorescence was not proportional to the size of the caries lesion. This result may indicate the inability of the DIAGNOdent to differentiate between superficial and deep dentinal caries as also indicated by other studies. ${ }^{10,11}$ The angulation of the DIAGNOdent tip and the possible presence of residues even after prophylaxis also may have affected the readings.

The sensitivity and specificity of DIAGNOdent for the detection of dentinal lesions on occlusal surfaces were calculated at 0.83 and 0.60 for the cutoff value of 20 ("initial caries lesion on dentin" according to the manufacturer), and at 0.66 and 0.72 for the cutoff value of 30 
("advanced caries lesion on dentin" also according to the manufacturer). These results were comparable with previous values reported by Lussi and Francescut ${ }^{25}(0.75$ and $0.68)$, Heinrich-Weltzien et al. ${ }^{26}$ (0.84 and 0.70), Angnes et al. ${ }^{27}$ (0.81 and 0.54), Cortes et al. ${ }^{28}$ (0.84 and 0.67), and AlwasDanowska et al. ${ }^{17}$ (0.95 and 0.52). However, the results of this study did not agree with the values reported by Lussi et al., ${ }^{2}$ who originally established these cutoffs for DIAGNOdent. It is interesting to note that the sensitivity of DIAGNOdent decreased when the cutoff was set at 30 . This fact also may corroborate the inability of the device to accurately diagnose deeper dentinal caries.

The poor specificity of the DIAGNOdent readings, as demonstrated in this and other studies, has important implications for clinicians. Low specificity results in an increased likelihood of falsepositive diagnoses and leads the clinician to intervene restoratively in a surface that may actually not be carious. As demonstrated recently by Pereira et al., ${ }^{29}$ the use of adjunct caries detection devices such as DIAGNOdent, electrical conductance, and quantitative light-induced fluorescence does not improve the accuracy of examiners in detecting early occlusal caries lesions, but may lead them to overtreat.
The accuracy and precision of the method used in this study should be further investigated. Volume determination using composite resin should be assessed by different evaluators and compared with other methods such as computed tomography. Furthermore, in vivo assessment of diagnostic performance and longitudinal examination of the correlation between variation in DIAGNOdent readings and caries extension should be carried out.

Within the limitations of this study, it is possible to conclude that laser fluorescence measured with DIAGNOdent does not correlate well with caries extension. In other words, higher DIAGNOdent readings may not necessarily represent increasingly advanced caries into dentin. Consequently, therapy guidelines proposed based on the DIAGNOdent readings may not be valid. Although the sensitivity of DIAGNOdent for the detection of dentinal lesions on occlusal surfaces was high, the considerable likelihood of unnecessary treatment (false-positive results) may preclude the use of DIAGNOdent as a primary method of caries diagnosis.

DISCLOSURE AND ACKNOWLEDGMENTS

The authors of this research article have no personal or financial affiliation with any company producing the products used in this study that might be considered a conflict of interest in performing this research.

The authors would like to thank Dr. Lee Boushell, Dr. Georgia Macedo, Dr. Vishnu Raj, and Dr. Gustavo Oliveira for their assistance in this study, and $\mathrm{KaVo}$ Dental Corporation for donating the DIAGNOdent device used in this study.

\section{REFERENCES}

1. Mjor IA. Clinical diagnosis of recurrent carries. J Am Dent Assoc 2006;136:1426-33.

2. Lussi A, Megert B, Longbottom C, et al. Clinical performance of a laser fluorescence device for detection of occlusal caries lesions. Eur J Oral Sci 2001; 109:14-9.

3. Attrill DC, Ashley PF. Occlusal caries detection in primary teeth: a comparison of DIAGNOdent with conventional methods. Br Dent J 2001;190:440-3.

4. Wenzel A, Larsen MJ, Fejerskov O. Detection of occlusal caries without cavitation by visual inspection, film radiographs, xeroradiographs, and digitized radiographs. Caries Res 1991;25:365-71.

5. Bader J, Shugars D. Variation in dentists' clinical decisions. J Public Health Dent 1995;55:181-8.

6. Bader JD, Shugars DA, Bonito AJ. A systematic review of the performance of methods for identifying carious lesions. J Public Health Dent 2002;62(4):201-13.

7. McComb D, Tam LE. Diagnosis of occlusal caries: Part I. Conventional methods. J Can Dent Assoc 2001;67: 454-7.

8. Pretty IA. Caries detection and diagnosis: novel technologies. J Dent 2006;34:72739.

9. Jeon RJ, Han C, Mandelis A, et al. Diagnosis of pit and fissure caries using 
frequency-domain infrared photothermal radiometry and modulated laser luminescence. Caries Res 2004;38:497-513.

10. Emami Z, al-Khateeb S, de Josselin de Jong E, et al. Mineral loss in incipient caries lesions quantified with laser fluorescence and longitudinal microradiography. A methodologic study. Acta Odontol Scand 1996;54:8-13.

11. al-Khateeb S, ten Cate JM, AngmarMansson B, et al. Quantification of formation and remineralization of artificial enamel lesions with a new portable fluorescence device. Adv Dent Res 1997; $11: 502-6$.

12. Shi XQ, Welander U, Angmar-Månsson B. Occlusal caries detection with $\mathrm{KaVo}$ DIAGNOdent and radiography: an in vitro comparison. Caries Res 2000;34: $151-8$.

13. Hafström-Bjrköman U, Sundström F, de Josselin de Jong E, et al. Comparison of laser fluorescence and longitudinal microradiography for quantitative assessment of in vitro enamel caries. Caries Res 1992;26:241-7.

14. Tranaeus S, Lindgren LE, Karlsson L, Angmar-Mansson B. In vivo validity and reliability of IR fluorescence measurements for caries detection and quantification. Swed Dent J 2004;28:173-82.

15. Heinrich-Weltzien R, Kuhnisch J, Oehme $\mathrm{T}$, et al. Comparison of different DIAGNOdent cut-off limits for in vivo detection of occlusal caries. Oper Dent 2003;28(6):672-80.

16. Ouellet A, Hondrum SO, Pietz DM. Detection of occlusal carious lesions. Gen Dent 2002;50:346-50.
17. Alwas-Danowska HM, Plasschaert AJ, Suliborski S, Verdonschot EH. Reliability and validity issues of laser fluorescence measurements in occlusal caries diagnosis. J Dent 2002;30:129-34.

18. Hamilton JC, Gregory WA, Valentine JB. DIAGNOdent measurements and correlation with the depth and volume of minimally invasive cavity preparations. Oper Dent 2006;31:291-6.

19. Bader JD, Shugars DA. A systematic review of the performance of a laser fluorescence device for detecting caries. J Am Dent Assoc 2004;135:1413-26.

20. Ismail AI, Sohn W, Tellez M, et al. The International Caries Detection and Assessment System (ICDAS): an integrated system for measuring dental caries. Community Dent Oral Epidemiol 2007; 35:170-8.

21. Francescut P, Zimmerli B, Lussi A. Influence of different storage methods on laser fluorescence values: a two-year study. Caries Res 2006;40:181-5.

22. Banerjee A, Kidd EA, Watson TF. In vitro evaluation of five alternative methods of carious dentine excavation. Caries Res 2000;34:144-50.

23. Dommisch $\mathrm{H}$, Peus $\mathrm{K}$, Kneist $\mathrm{S}$, et al. Fluorescence-controlled Er:YAG laser for caries removal in permanent teeth: a randomized clinical trial. Eur J Oral Sci 2008;116:170-6.

24. Humphrey SP, Williamson RT. A review of saliva: normal composition, flow, and function. J Prosthet Dent 2001;85:162-9.

25. Lussi A, Francescut P. Performance of conventional and new methods for the detection of occlusal caries in deciduous teeth. Caries Res 2003;37:2-7.

26. Heinrich-Weltzien R, Kuhnisch J, Oehme $\mathrm{T}$, et al. Comparison of different DIAGNOdent cut-off limits for in vivo detection of occlusal caries. J Oral Rehabil 2004;31:895-9.

27. Angnes V, Angnes G, Batisttella M, et al. Clinical effectiveness of laser fluorescence, visual inspection and radiography in the detection of occlusal caries. Caries Res 2005;39:490-5.

28. Cortes DF, Ellwood RP, Ekstrand KR An in vitro comparison of a combined FOTI/visual examination of occlusal caries with other caries diagnostic methods and the effect of stain on their diagnostic performance. Caries Res 2003;37:8-16

29. Pereira AC, Eggertsson H, Martinez-Mier EA, et al. Validity of caries detection on occlusal surfaces and treatment decisions based on results from multiple cariesdetection methods. Eur J Oral Sci 2009;117:51-7.

Reprint requests: André V. Ritter, DDS, MS, UNC Chapel Hill, School of Dentistry, 433 Braner Hall, Chapel Hill, NC, USA 27599; Tel: (919) 843-6356; email: rittera@ dentistry.unc.edu

This article is accompanied by commentary, "Correlation Between Laser Fluorescence Readings and Volume of Tooth Preparation in Incipient Occlusal Caries in Vitro," Joel Berg, DDS, MS, DOI 10.1111/j.17088240.2009.00310.x 